\title{
Drunk Driving Detection
}

\author{
Kavish Atul Sanghvi \\ Mukesh Patel School of Technology, Management and Engineering, India
}

Copyright $(2018$ by authors, all rights reserved. Authors agree that this article remains permanently open access under the terms of the Creative Commons Attribution License 4.0 International License

\begin{abstract}
Drunk driving accidents have increased day by day and has become a big issue. On an average nearly $29 \%$ of road accidents are caused due to drunk driving. To avoid such accidents, precautionary measures using different technologies are taken. Drunk driving detection is process of detecting whether a person is drunk or not. It uses data provided by sensors and camera to detect whether the person is drunk or not. The data is further processed using specific algorithm and methods to detect whether person is drunk or not. It uses various detection methods like Iris recognition using Gabor filter, Neural network using face images, detection using speech, Non-invasive Biological sensors, detection using Driving pattern and Engine locking system.
\end{abstract}

Keyword Gabor Filter, Neural Network, Biological Sensor

\section{Introduction}

In the modern day, with the increase in the number of vehicles plying on the roads, traffic accidents have grown significantly in number. One of the primary causes of traffic accidents is drunk driving or driving under influence (DUI). This is particularly an important issue for developing countries, such as India, where $53.4 \%$ of unnatural deaths in the year 2014 were due to traffic accidents, with drunk driving being the primary cause [1]. Currently, police inspecting roads sample cars for breath tests to detect alcohol levels. However, this approach is manual and unlikely to detect most cases of driving under influence of alcohol. Alternate and more effective approaches to detect drunk driving may include automatic detection using sensors. Prevention may include reducing the car's speed or alerting people or police via the internet. The network of physical devices embedded with sensors, electronics, computing modules and network connectivity using which they aggregate and share data. In this specific case, sensors to detect alcoholism can be directly or indirectly used. This can be connected to mobile phones which contain communication network for appropriately alerting people. Several models can be found in literature. However, no specific model has been adopted for widespread use. The various methods proposed face problems such as difficulty in implementation, scalability and complexity. Therefore, new models need to maintain cheap cost, easy implementation along with accuracy.

\section{Related Works}

\section{A. Drunk Driving Detection through Iris Recognition Using Gabor Filter}

The alcohol detection system through Iris recognition using Gabor filter, it is focused on three main tasks. First is capturing an iris image [2]. Secondly, it is necessary to encode the iris information into a format which is responsive to calculation and computation. Finally, a signal coming from the open source recognition system will manipulate the car/vehicle through the use of microcontroller and relay circuit which is connected to the ignition system of the car/vehicle. Once the driver is detected under the influence of alcohol, the ignition system will be locked down. To identify the difference between the size of pupil before and after alcohol consumption, a computation was designed

$$
\frac{p_{\text {re }}-p_{\text {ost }}}{\text { Pre }} * 100
$$




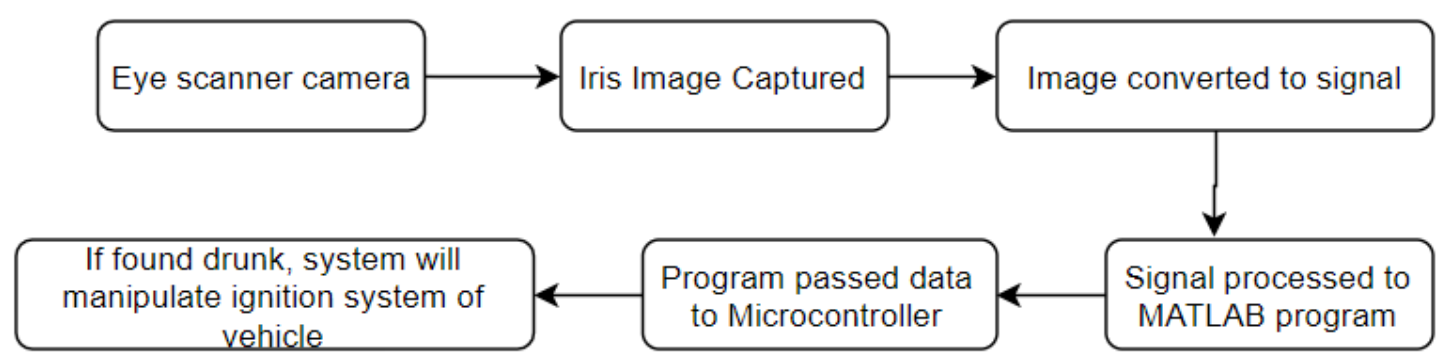

Figure 1. Block diagram of Iris Recognition using Gabor filter

\section{B. Drunk Driving Detection}

Drunk driving detection using neural networks uses a camera for capturing face images [3]. The images are captured not only while turning on the engine but also while driving to avoid cheating in detection. A 3-layered fully connected neural network system is used to detect whether a person is drunk or not. Neural network is trained with the back-propagation method by giving face images before and after drinking and examine the accuracy of detection by applying test data. The back-propagation algorithm updates the weight from the output layer to the input layer in reverse order. The process is repeated until the error energy is smaller than threshold.

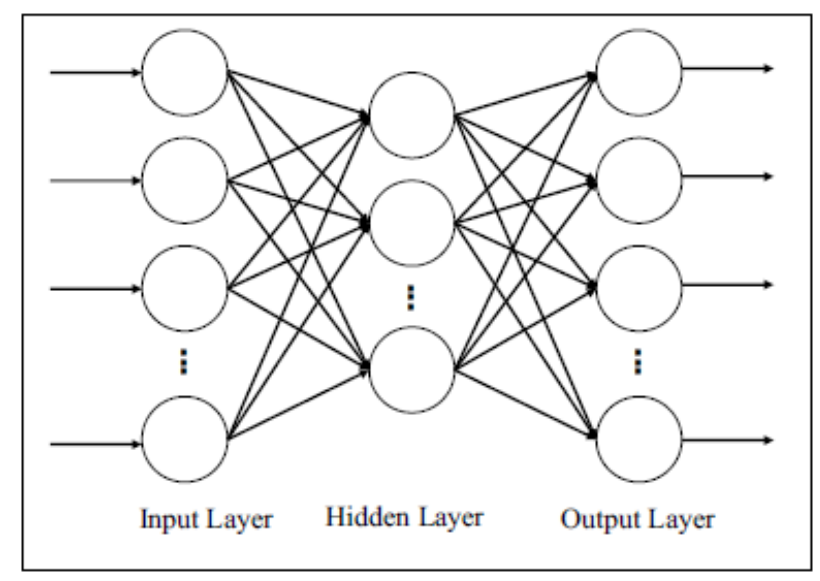

Figure 2. A 3-layered neural network

The RGB value of face images is used. A small part from the face image is cut out and resizes it in small size. The RGB values are normalized and are provided to the input layer of the neural network. The output layer indicates whether person is drunk or not. The cheek image of size $5 * 5$ extracted from images is provided as input to the neural network. The output layer is composed of 2 units, namely u1: drinking and u2: no drinking. Cheek portion is extracted from the face image captured from camera, this image is resized into 5 lines $* 5$ pixels and convert the image into three images corresponding to each of RGB components. Finally, normalize the values to $[0,1]$ to use as input to the neural network.

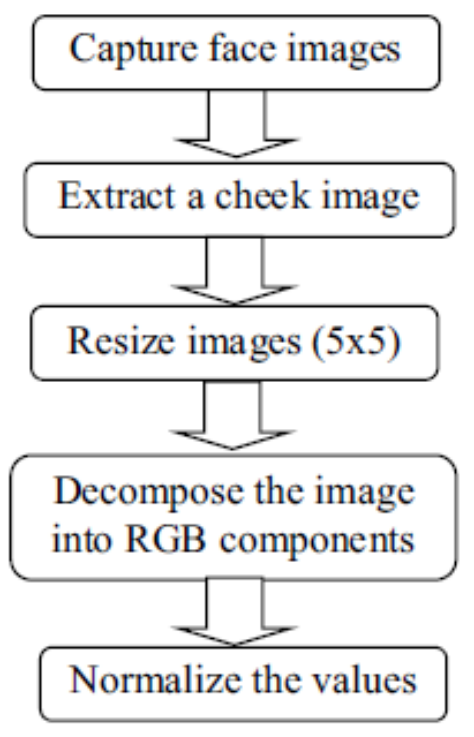

Figure 3. Flow diagram of extracting input data from face image

Rule obtained to decide whether drunk or not

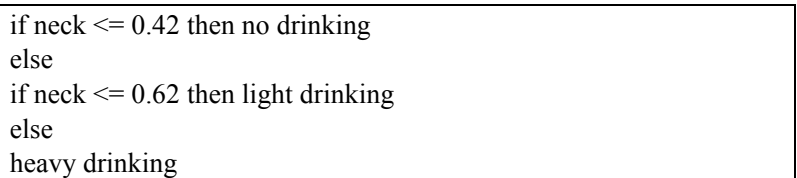

The accuracy of the face images is calculated using following formula

Accuracy $=\frac{\text { Number of classification image }}{\text { Number of test images }} * 100$ 
Table 1. Color chart for drunk level

\begin{tabular}{|c|c|c|c|c|c|c|}
\hline \multirow[b]{2}{*}{ Name } & \multirow{2}{*}{$\begin{array}{l}\text { Drink- } \\
\text { ing }\end{array}$} & \multicolumn{5}{|c|}{ Parts used for classification } \\
\hline & & cheek & chin & neck & ear & hand \\
\hline $\mathrm{N}$ & no & & & & & \\
\hline 0 & no & & & & & \\
\hline I & no & & & & & \\
\hline P & no & & & & & \\
\hline Q & no & & & & & \\
\hline M & light & & & & & \\
\hline D & light & & & & & \\
\hline 0 & light & & & & & \\
\hline P & light & & & & & \\
\hline E & light & & & & & \\
\hline $\mathrm{R}$ & heavy & & & & & \\
\hline D & heavy & & & & & \\
\hline P & heavy & & & & & \\
\hline E & heavy & & & & & \\
\hline I & heavy & & & & & \\
\hline
\end{tabular}

\section{Drunk Driving Detection Using Speech}

A traditional approach to speaker state detection includes two states: the extraction of low-level acoustic features, and a n-way direct classification or regression using maximum margin classifiers [4]. Drunk speaker may use prosodic contours differently from sober speaker. Energetic drunk speaker may systematically more emphasize than sober speaker, whereas depressed drunk speaker might use less emphasis. Phasing is thought to be influenced by sentence planning, if a speaker's ability to plan future constituents is impaired by alcohol, they may include more disfluenced and intonational phrase boundaries. AuToBI toolkit is used to identify prosodic events automatically. Intoxication may cause speakers to pronounce words differently, choosing certain pronunciation variants more frequently than others, and may even choose certain words more frequently affecting the phonotactic patterns in each class [5]. It is useful to report detection error tradeoff (DET) curve, which plots false alarm vs. miss probabilities of missing intoxicated speaker. The DET curve determines the detection threshold.

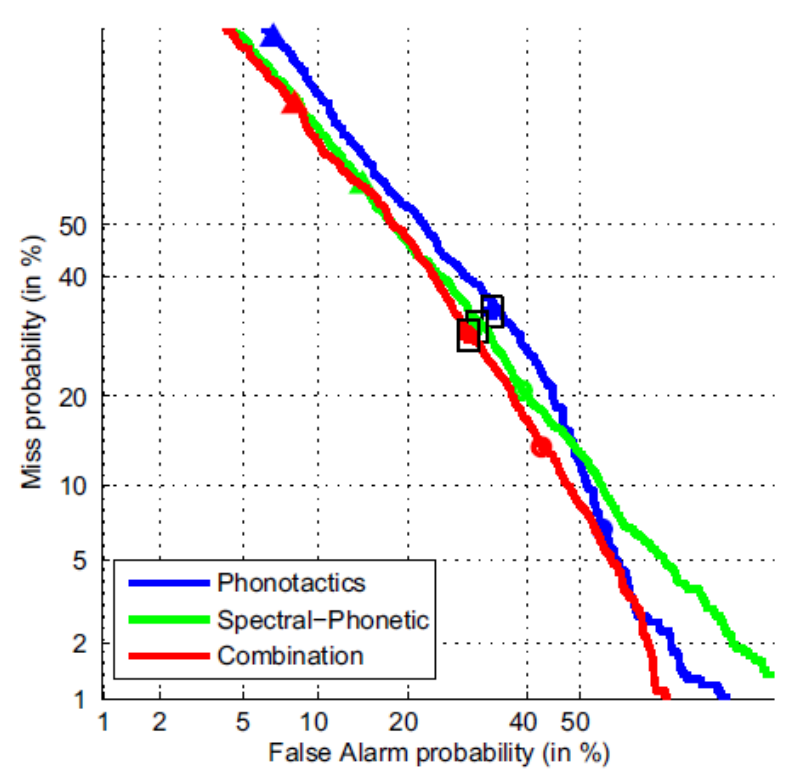

Figure 4. DET of official development set

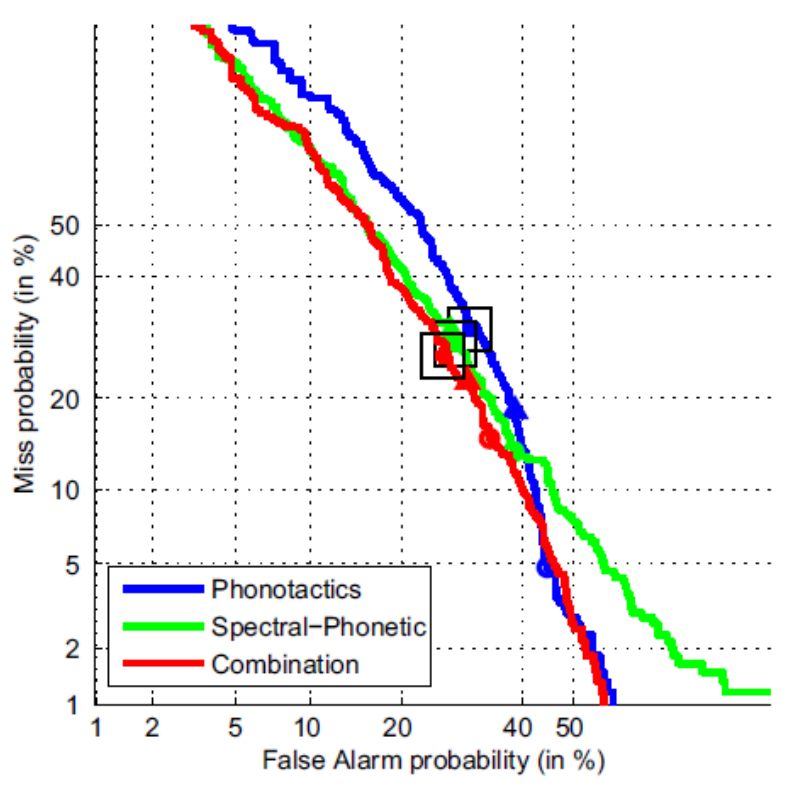

Figure 5. DET of balanced set

\section{Non-invasive Biological Sensor System for Detection of Drunk Driving}

A seat containing the air-pack sensor used to monitor the AP-PW is shown in Fig. 6. The digital pulse volume, using a finger clip photoplethysmography and the breath-alcohol concentration were measured with AP-PW simultaneously [6]. Four subjects were healthy adults (Subject A, B and C were males and subject $D$ was female) with mean age of 21 
years. Biological signals were taken from the subjects 20 minutes prior to consuming alcohol, for subsequent comparison to signals from the non-invasive sensors in the air pack. Recorded biological data was $200 \mathrm{~Hz}$. Subjects then consumed alcohol (Beer, $500 \mathrm{ml}$ ) within 10 mins. The first measurement was taken during a 20 mins period 20-40 minutes after consumption of alcohol, when the blood alcohol is believed to reach highest levels. To observe the changes over time in all subjects, measurements were also recorded at 90-110 minutes and 160-180 minutes, for total of four measurements. Subjects had not eaten for at least 3 hours prior to consuming the alcohol, but we provided a typical volume of snacks along with the alcohol, to reproduce usual conditions under which alcohol is consumed. The breath-alcohol concentration was measured before and after measuring all biological signals.
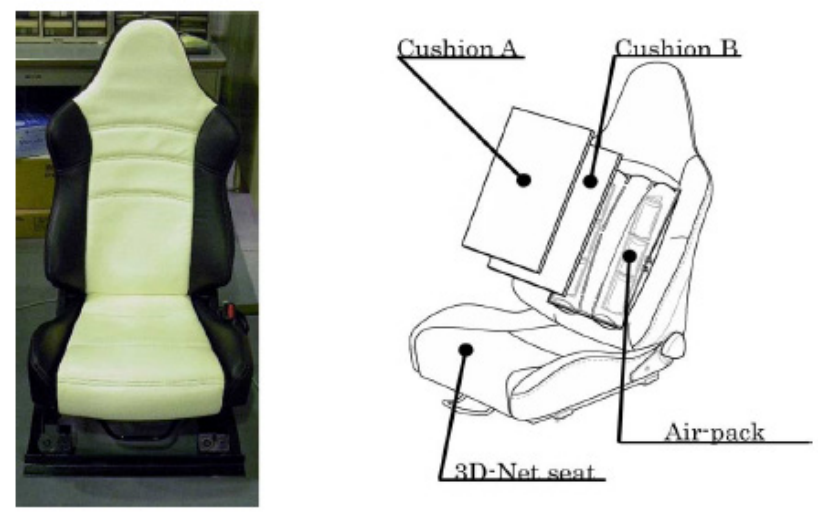

Figure 6. Experimental apparatus

The original waveforms from data strings of the digital pulse volume and the AP-PW from the first 5 minutes of measurements in each period for all subjects were compared using the FFT analysis. The analysis of the time series for frequency fluctuation of the AP-PW curves, in Fig. 7. Indicate methods used in the calculation. The AP-PW was filtered using frequency analysis to separate the signal of the body-trunk plethysmogram. The Savitzky and Golay smoothing filter is used to find maximum value in the time series of the AP-PW data.

Breath-alcohol concentration was measured before and after drinking as shown in Fig. 8. For all subjects, the breath-alcohol concentration increased more than 0.1 $\mathrm{mg} / \mathrm{dl}$ to an invigorated state.

\section{E. Drunk Driving Detection Using Driving Pattern}

Drunk driving detection using driving pattern uses mobile phone as the platform for drunk detection as they combine the detection and communication functions [7]. As a self-contained device, mobile phone presents a mature hardware and software environment for the development of active drunk driving monitoring system. The system based on mobile phone can function effectively on its own because mobile phones are highly portable, all necessary components are already integrated, and their communication services have vast coverage. The minimum requirement for such a mobile phone platform is the presence of simple sensors like accelerometer, orientation sensor, etc. The communication module and speaker are good enough for alerting. Drunk driving related behavior are classified into three categories. The first and second category focus on driving behaviors related to vehicle movement; the third category is about driving behavior related to subjective judgment and vigilance of the driver.

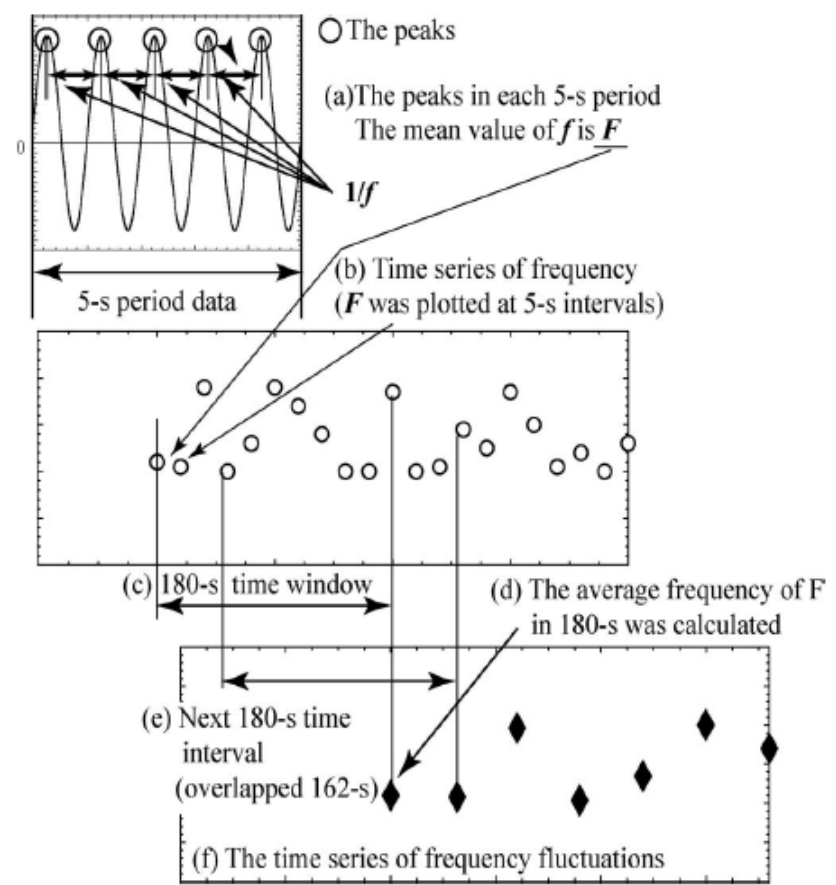

Figure 7. Time series computation of frequency fluctuation

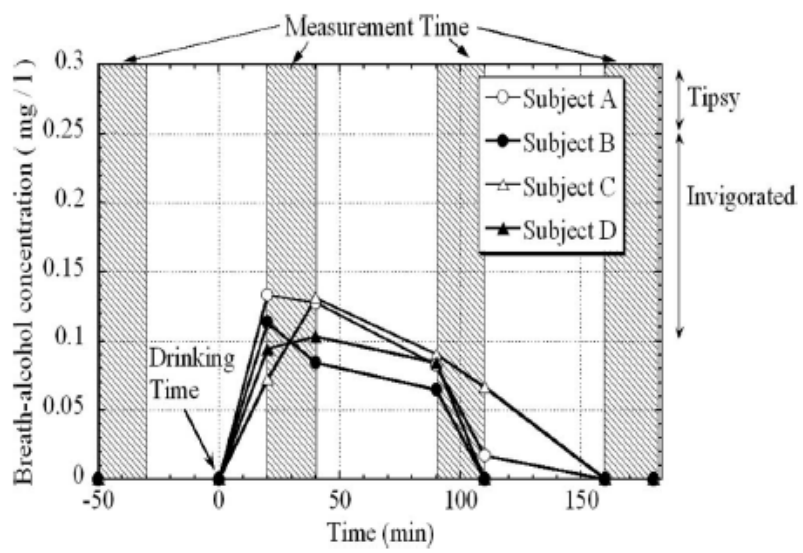

Figure 8. Breath - alcohol concentrations of the subjects 


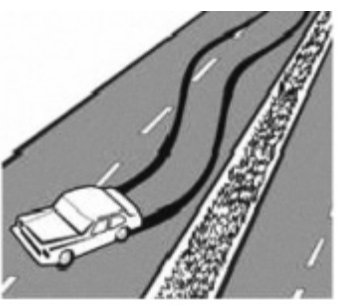

(a)

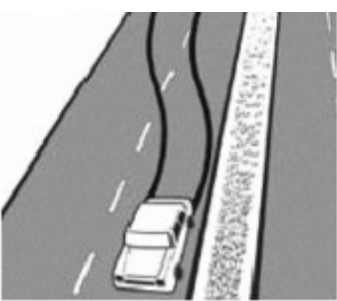

(b)

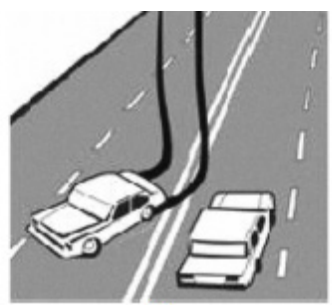

(c)

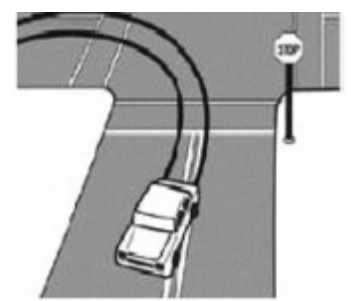

(d)

Figure 9. Problems in maintaining the lane position: (a) weaving, (b) drifting, (c) swerving, (d) turning with a wide radius

The system includes following modules: (1) monitoring daemon module, (2) calibration module, (3) data processing and pattern matching module and (4) alert module. The third module implements the detection algorithm, as marked by a dashed box. A calibration procedure is conducted when system detects phone is located in a moving vehicle; then the main program is launched, working as a background daemon [8]. The daemon monitors the driving pattern and behavior in real time and collects acceleration information. The collected information includes lateral and longitudinal acceleration. They are processed separately, and used as inputs to the multiple round pattern matching process. If the pattern condition is satisfied, which means drunk driving is detected; one signal is transmitted to trigger an alert. The phone alarms to remind the driver and automatically contact the police station. If the condition is not satisfied, execution returns to the daemon immediately.

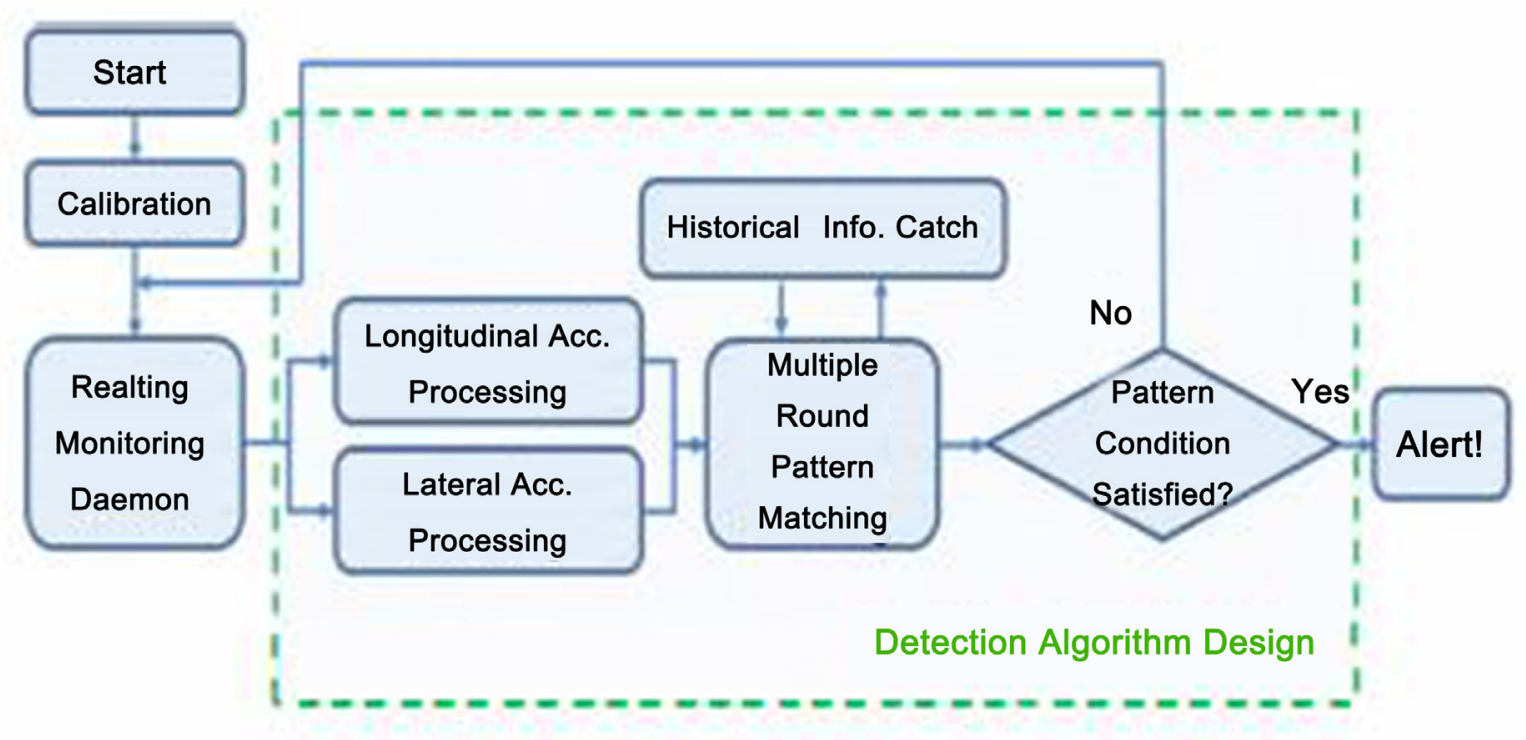

Figure 10. Working procedure for drunk detection system

\section{F. Drunk Driving Detection Based on Engine Locking System}

Drunk driving detection based on Engine locking system is used to detect whether a person is drunk or not and if the person is drunk, the system prevents them from driving and notifies detection of alcoholic driver to nearest police station. This system monitors behavior of vehicle both inside and outside of the vehicle [9]. This system also notifies ambulance and relatives of driver incase accident occurs. 


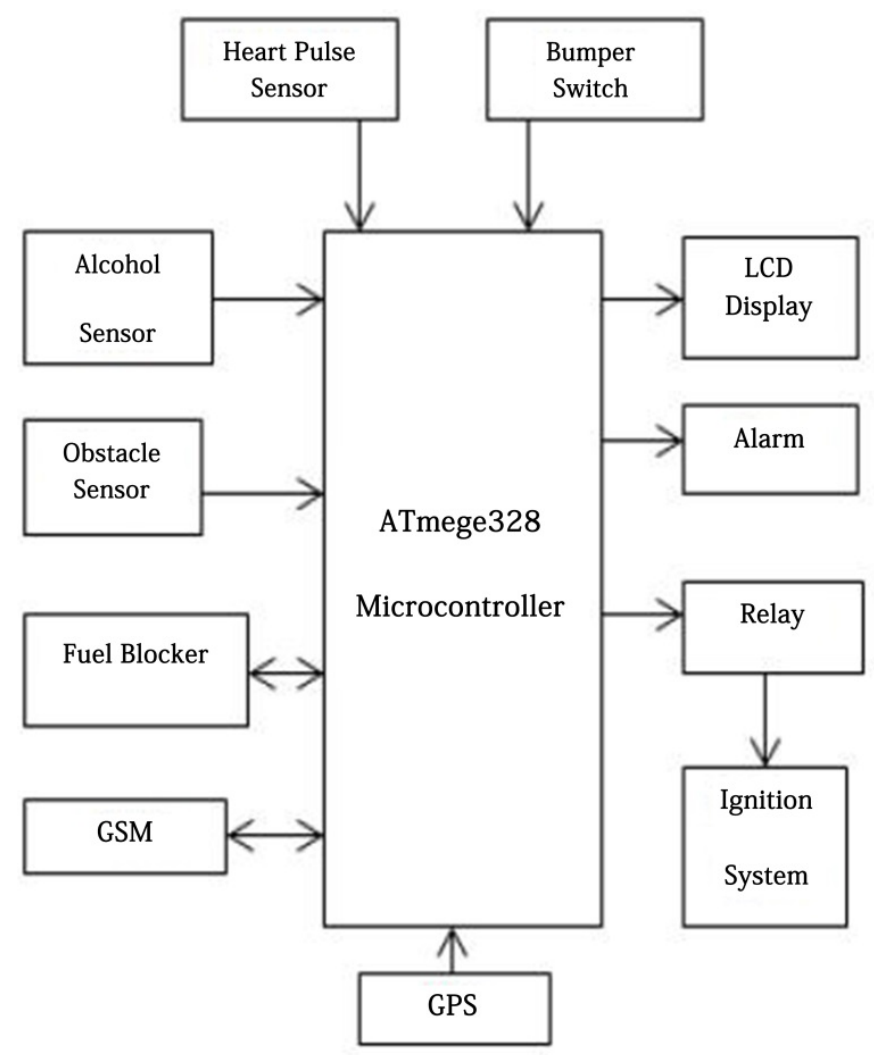

Figure 11. Block diagram

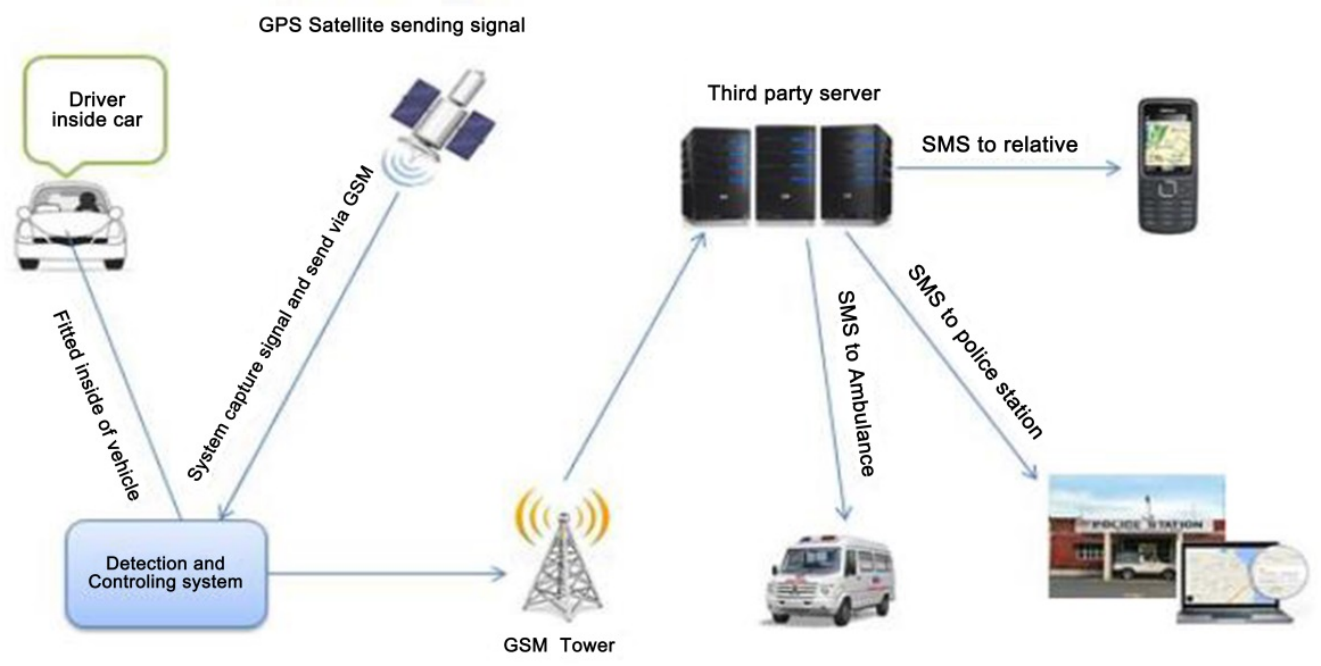

Figure 12. Architecture of the system

When driver starts vehicle, alcohol sensor (MQ-3) starts sensing vehicle speed equal to zero. If driver is detected drunk then immediately ignition system will turn off along alarm and notification to police station. A flag is set when first condition is passed without detection of alcohol. When speed of vehicle is greater than zero i.e. vehicle is in motion, along with alcohol sensor start sensing and send collected parameter values to microcontroller. If detected drunk in this case, then signal is send to fuel blocker by microcontroller for blocking fuel supply to ignition system.
Also, it alerts driver by alarm and vehicle is stopped at that location along with notification to police station.

\section{Modifications}

Various cheap sensors are available for detection of alcohol levels. However, it is clear that solely relying on such sensors is not an effective solution to the drunken driving problem. Therefore, a holistic method is required to 
combine a number of factors and personalize for specific people, instead of a generalized approach.

The parameters considered are:

- Alcohol content

- Eye blink rate and facial recognition

- Acceleration and general movement of the car

- $\quad$ Nature of the road, i.e. likelihood of accidents

Alcohol content is the most fundamental detail required. It can be easily detected using a variety of sensors. The raw data from the alcohol sensor is passed and thresholded, depending on the alcohol levels. Eye blink rate is another parameter discussed in the model. As with alcohol content, sensors are available with this task. Facial recognition, in combination with techniques such as machine learning, can also be used to provide a score on how drowsy a person appears to be. These parameters can also trace other effects such as sleep deprivation. Acceleration and movement of the car is a parameter that significantly depends on the driver's specific nature. Therefore, classification approaches can be used to detect if a driver is taking abnormal decisions or life-threatening decisions.

\section{Conclusions}

Drunk driving accidents are an everyday affair. This paper has listed several different methods to analyze drunken driving, and also listed their advantages and shortcomings. It is seen that combining various approaches with statistical learning tools can provide exciting results in this field.

\section{REFERENCES}

[1] Suparna Sahabiswas, Sourav Saha, Prachatos Mitra,
Retabrata Chatterjee, Ronit Ray, Paramartha Saha, Rajarshi Basu, Saurav Patra, Pritam Paul, Bidrohi Ananya Biswas, "Drunk Driving detection and prevention models using Internet of things", in 2016 IEEE

[2] Lea Angelica Navarro, Mark Anthony Dino, Exechiel Joson, Rommel Anacan, Roberto Dela Cruz, "Design of Alcohol Detection System for Car Users thru Iris Recognition Pattern Using Wavelet Transform", in IEEE $20167^{\text {th }}$ International conference on Intelligent systems, modelling and simulation

[3] Kenichi Takahashi, Kana Hiramatsu, Mami Tetsuishi, "Experiments on Detection of Drinking from Face Images Using Neural Networks", in IEEE $20152^{\text {nd }}$ International conference on Soft Computing and Machine Intelligence

[4] Fadi Biadsy, William Yang Wang, Andrew Rosenberg, Julia Hirschberg, "Intoxication Detection using Phonetic, Phonotactic and Prosodic Cues", in ISCA, August 2011

[5] Matthew Marge, "Detecting Intoxication in Speech", in INTERSPEECH 2011

[6] Kohji Murata, Etsunori Fujita, Shigeyuki Kojima, Shinitirou Maeda, Yumi Ogura, Tsutomu Kamei, Toshio Tsuji, Shigehiko Kaneko, Masao Yoshizumi, Nobutaka Suzuki, "Noninvasive Biological Sensor System for Detection of Drunk Driving", in IEEE Transactions on Information Technology in Biomedicine, Vol. 15, No. 1, January 2011

[7] Jiangpeng Dai, Jin Teng, Xiaole Bai, Zhaohui Shen, Dong Xuan, "Mobile Phone Based Drunk Driving Detection", in IEEE $4^{\text {th }}$ International ICST Conference on Pervasive Computing Technologies, 2010

[8] Prarna Dhar, Sarika Shinde, Nikhil Jadav, Anirudha Bhaduri, "Unsafe Driving Detection System using Smartphone as Sensor Platform", in International Journal of Enhanced Research in Management \& Computer Applications, Vol. 3, Issue 3, Page 65-70, March 2014

[9] Rahul Mandalkar, Rahul Pandore, Manoj Shinde, Valmik Godse, "Alcohol Detection and Accident Avoidance Using Locking with Tracking", in International Journal of Advance Research in Computer Science and Management Studies, Vol. 3, Issue 9, September 2015. 